\title{
Nefrostomia percutânea ecoguiada em cães
}

\author{
Ultrasound-guided percutaneous nephrostomy in dogs
}

\author{
Ingrith Aparecida Mazuhy Santarosa' Carmen Lice Buchmann de Godoy ${ }^{\text {II }}$ \\ Ney Luis Pippi ${ }^{\mathrm{III}}$ Paulo Sérgio Pase Antunes ${ }^{\mathrm{IV}}$ Josaine Cristina da Silva Rappeti $^{\mathrm{I}}$ \\ Giovani Krolikowski ${ }^{I}$ Deisi Novosad ${ }^{V}$ Vanessa Sarturi Gheller ${ }^{\mathrm{VI}}$
}

\section{RESUMO}

A hidronefrose decorre freqüentemente da obstrução parcial ou completa do fluxo de urina e pode afetar um ou ambos os rins, causando dilatação da pelve $e$ comprometimento funcional progressivo. A drenagem urinária por nefrostomia percutânea promove a diminuição imediata da pressão intra-renal, desviando a urina de seu trajeto habitual, quando o mesmo encontra-se prejudicado. O objetivo deste estudo foi descrever e adequar a técnica de nefrostomia percutânea para seu uso em cães, utilizando a ultra-sonografia como guia em tempo real. O procedimento foi realizado em oito cães, submetidos à produção experimental de hidronefrose unilateral. As técnicas de Seldinger e de inserção direta foram utilizadas para a introdução de um cateter de modelo pigtail. A confirmação do cateter na pelve renal foi feita pela infusão de meio de contraste à base de iodo, através do mesmo, conforme a técnica de pielografia percutânea anterógrada. A nefrostomia percutânea ecoguiada demonstrou resultados satisfatórios na drenagem urinária de hidronefrose em cães, provando ser uma alternativa rápida, eficiente e segura.

Palavras-chave: hidronefrose, drenagem urinária percutânea, cateter pigtail.

\section{ABSTRACT}

Hydronephrosis often originates from the partial or complete obstruction of the urine flow and it can affect one or both kidneys, causing dilation and progressive functional impairment. Urinary drainage by percutaneous nephrostomy promotes immediate relief of intrarenal pressure, diverting the urine flow when its habitual trajectory is damaged. The aim of this study was to describe and adjust the percutaneous nephrostomy technique, using ultrasound as a real-time guide. The procedure was performed on eight dogs, with unilateral hydronephrosis produced experimentally. The Seldinger and direct insertion techniques were used for the introduction of a pigtail catheter. The confirmation of the insertion of the cateter into the kidney was carried out by the infusion of iodine contrast medium according to the percutaneous antegrade pyelography. The technique demonstrated satisfactory results in urinary drainage hydronephrosis in dogs, proving to be a quick, efficient and safe alternative.

Key words: hydronephrosis, percutaneous urinary drainage, pigtail catheter.

\section{INTRODUÇÃO}

A ultra-sonografia intervencionista referese aos procedimentos que utilizam a imagem fornecida pelo ultra-som, para guiar instrumentos como agulhas e cateteres até o interior dos tecidos. Atualmente, esse método tem sido utilizado como alternativa às intervenções cirúrgicas, nas chamadas técnicas minimamente invasivas (SOLBIATI, 1998). Em medicina veterinária, intervenções que utilizam a ultra-sonografia como guia em tempo real são indicadas para realização de biopsias, aspirações e drenagens percutâneas de lesões cavitárias (KANAYAMA, 2004).

Hidronefrose é o resultado da completa ou parcial obliteração do fluxo urinário que pode atingir

\footnotetext{
IPrograma de Pós-graduação em Medicina Veterinária, Universidade Federal de Santa Maria (UFSM). Endereço para correspondência: Rua Albion, 402/119B, Partenon, 91530-010, Porto Alegre, RS, Brasil. E-mail: ingsrosa@yahoo.com.br.

"Departamento de Clínica de Grandes Animais (DCGA), UFSM, Santa Maria, RS, Brasil.

IIIDepartamento de Clínica de Pequenos Animais (DCPA), UFSM, Santa Maria, RS, Brasil.

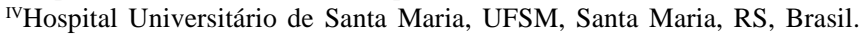

${ }^{\vee}$ Curso de Medicina Veterinária, UFSM, Santa Maria, RS, Brasil.

${ }^{\mathrm{V}}$ Autônomo, Santa Maria, RS, Brasil.
} 
um ou ambos os rins. A obstrução causa distensão progressiva da pelve e, conseqüentemente, atrofia parenquimal (BERCOVITCH, 2000), sendo que o grau da lesão depende da integridade e do tempo de obstrução (CHRISTIE \& BJORLING, 1998; McLOUGHLIN, 2000).

A nefrectomia é indicada nos casos de hidronefrose avançada, quando o rim se apresenta como uma saculação repleta de líquido (CHRISTIE \& BJORLING, 1998) ou frente às afecções renais e ureterais que desafiem o reparo cirúrgico (FOSSUM, 2005). Quando a urografia excretora (CHRISTIE \& BJORLING, 1998) e/ou a cintilografia (HUDSON et al., 2003) evidenciarem alguma função renal, a recuperação do rim passa a ser fundamental, especialmente se o outro estiver lesionado (HARDIE \& KYLES, 2004). O comprometimento total da função renal nos cães portadores de uropatia obstrutiva pode ser evitado, promovendo-se o alívio da pressão intra-renal (CHRISTIE \& BJORLING, 1998). A drenagem urinária por nefrostomia permite desviar a urina para o meio externo através da instalação de uma sonda no interior da pelve renal, por meio de laparotomia (CHRISTIE \& BJORLING, 1998; HARDIE \& KYLES, 2004; FOSSUM, 2005) ou através de uma incisão abdominal, localizada próxima ao rim (NWADIKE et al., 2000).

Animais com obstrução ureteral, freqüentemente associada à disfunção renal, podem ser previamente estabilizados por manobras clínicas associadas a procedimentos menos invasivos como a nefrostomia percutânea ecoguiada, antes de serem submetidos aos tratamentos que removem a obstrução (HARDIE \& KYLES, 2004). Além disso, a instalação de um cateter de nefrostomia no período pré-operatório permite a avaliação da função restante do rim obstruído, favorece a diurese fisiológica antes do reparo definitivo (NWADIKE et al., 2000) e fornece tempo para o planejamento de uma terapia adequada (ROVEN \& ROSEN, 1984).

Para nefrostomia percutânea em humanos, técnicas de imagem como a ultra-sonografia, a fluoroscopia (STABLES, 1982; THÜROFF, 1988; GUPTA et al., 1998; EARP, 2000; WAH et al., 2004) ou a tomografia computadorizada são requeridas para a inserção do cateter no rim (STABLES, 1982; THÜROFF, 1988). Este pode ser mantido no interior da pelve renal até que se tenha corrigido a causa da obstrução, ou por período indeterminado de tempo (EARP, 2000; WAH et al., 2004). O procedimento, quando realizado apenas sob controle ecográfico, dispensa o uso de meio de contraste intravenoso, necessário na fluoroscopia e contra-indicado em alguns pacientes (STABLES, 1982; ROVEN \& ROSEN, 1984).
O preparo prévio do paciente inclui exame laboratorial, estando contra-indicada a técnica em pacientes portadores de coagulopatias (THÜROFF, 1988; WAH et al., 2004). As drenagens percutâneas são recomendadas para pacientes de alto risco e casos de recorrência ou pobre resposta ao tratamento (PENNINCK, 1995).

A escolha da área de introdução do cateter pode variar de acordo com o tamanho do paciente, a posição do rim e dos órgãos adjacentes (THÜROFF, 1988). Para que o instrumento seja visibilizado por completo, deve estar localizado dentro do feixe sonoro, inserido num ângulo de aproximadamente $45^{\circ}$ em relação ao transdutor e alinhado à linha mediana do mesmo, segundo a técnica à mão livre, rotineiramente utilizada em procedimentos percutâneos ecoguiados (KANAYAMA, 2004; NYLAND et al., 2005). A ultrasonografia permite medir a profundidade e a rota da punção (EARP, 2000), mostrando a pelve dilatada e o instrumento num mesmo plano ecográfico (THÜROFF, 1988).

A rota do percurso deve ser estabelecida de modo que, antes de penetrar na pelve, a agulha passe através do parênquima renal, que atua como um tampão, prevenindo vazamento de urina (LANG \& PRICE, 1983). Dessa forma, evita-se também lesão na vasculatura do hilo renal (STABLES, 1982). A indesejável perfuração de órgãos como intestino e baço, durante a realização de procedimentos percutâneos, pode ser evitada aplicando-se firme pressão ao transdutor (NYLAND et al., 2005).

De acordo com o grau de complexidade do procedimento, seleciona-se o tipo de cateter para nefrostomia (PAUL et al., 2003). Dentre os diversos modelos, o pigtail é o mais comumente utilizado em humanos (EARP, 2000) e é mais bem aplicado quando utilizado para infusões terapêuticas intra-renais, após cirurgias renais pouco complexas e em drenagem temporária de urina. Sua extremidade distal possui formato helicoidal, mantido por memória, que, associado ao seu mecanismo de trava ou autobloqueio, previne deslocamentos (PAUL et al., 2003). Conforme EARP (2000), a adequada fixação do cateter à pele, mantendo-se alguma folga em pacientes humanos obesos, evita sua remoção inadvertida. Para nefrostomia percutânea ecoguiada, são utilizadas tanto a técnica de Seldinger, quanto a inserção direta (SZATMÁRI et al., 2001).

A técnica de Seldinger foi inicialmente desenvolvida para cateterização arterial percutânea, auxiliada por fio guia (SELDINGER, 1953). A partir daí, está sendo rotineiramente utilizada em humanos, para procedimentos como a nefrostomia (ROVEN \& ROSEN, 
1984; GUPTA et al., 1998; LEWIS \& PATEL, 2004) e é aplicada preferencialmente em casos de sistema coletor renal pouco ou não dilatado (WAH et al., 2004). Em cães, a técnica é descrita por SZATMÁRI et al. (2001) para introdução de cateter em pacientes com pionefrose. A inserção direta é recomendada para drenagem urinária temporária nos casos em que haja dilatação pélvica. Nessa técnica ecoguiada, o cateter e seus acessórios são inseridos diretamente no interior da pelve renal (WAH et al., 2004). A sonda é suturada à pele (ROVEN \& ROSEN, 1984) e uma bolsa urinária em sistema fechado é conectada à mesma, permitindo o fluxo livre de urina (EARP, 2000; WAH et al., 2004).

A pielografia anterógrada consiste na administração percutânea de meio de contraste diretamente no rim para promover a opacificação da pelve e ureter (MATTOON et al., 2005), verificando dessa forma o adequado posicionamento do cateter, seguido do procedimento de nefrostomia (ROVEN \& ROSEN, 1984). A infusão de meio de contraste, quando diluído a 30\%, permite uma maior visualização de detalhes da arquitetura renal (THÜROFF, 1988), não impedindo a visibilidade do instrumento de drenagem urinária inserido no rim (EARP, 2000). A quantidade de contraste a ser injetada equivale à metade do conteúdo puncionado (RIVERS et al., 1997). Radiografias em diferentes projeções são realizadas imediatamente após a infusão (MATTOON et al., 2005).

Em pacientes humanos, são relatadas complicações como hemorragia persistente, septicemia, lesão pélvica com extravasamento retroperitoneal de urina (LEWIS \& PATEL, 2004), perfuração de vísceras adjacentes e deslocamento do cateter (WAH et al., 2004). Um período transitório de hematúria freqüentemente ocorre imediatamente após a inserção do instrumento de nefrostomia, não persistindo por mais de 36 horas. Lavagens freqüentes do cateter com solução salina estéril previnem a interrupção do fluxo urinário causado por coágulos durante este período (STABLES, 1982).

O presente estudo teve a finalidade de adequar a técnica de nefrostomia percutânea para seu uso em cães, utilizando a ultra-sonografia como guia em tempo real para inserção de um cateter de modelo pigtail. Assim, descreve-se o procedimento, tendo em vista a escassez de literatura relacionada à medicina veterinária e sua incorporação à rotina, como mais uma alternativa a ser aplicada nos casos de uropatias obstrutivas que cursam com hidronefrose em cães.

\section{MATERIAL E MÉTODOS}

Foram utilizados oito cães, seis fêmeas e dois machos, adultos, sem raça definida, clinicamente saudáveis e com peso corporal entre 10 e 17,5kg, oriundos do Biotério Central da Universidade Federal de Santa Maria (UFSM). A seleção dos animais fundamentou-se no exame físico, na dosagem de creatinina sérica, na contagem plaquetária e posterior avaliação ultra-sonográfica do sistema urinário. Após um período de adaptação ao ambiente e vermifugação, os cães foram encaminhados ao bloco cirúrgico objetivando a ligadura unilateral do ureter para a produção de hidronefrose. A escolha do rim a ser obstruído foi aleatória, sendo que quatro cães tiveram o ureter direito ligado e quatro cães o ureter esquerdo. Exames ultra-sonográficos foram realizados a cada três dias para controle da hidronefrose. A drenagem urinária foi realizada no sétimo dia após a ligadura do ureter, com exceção de um cão que apresentou dilatação significativa da pelve renal apenas no $14^{\circ}$ dia pósligadura. Os exames e procedimentos ultrasonográficos foram realizados com aparelho de ultrasom SDU-395A $\mathrm{A}^{\mathrm{a}}$, utilizando transdutor setorial de $7,5 \mathrm{MHz}^{\mathrm{a}}$.

O preparo que antecedeu a nefrostomia percutânea ecoguiada incluiu ampla tricotomia da região paralombar direita ou esquerda e jejum alimentar de 12 horas. Os cães foram submetidos à anestesia geral, sendo que o protocolo instituído foi semelhante em todos os procedimentos. O posicionamento utilizado foi o decúbito lateral, com o lado correspondente ao rim a ser drenado voltado para cima. Um suporte feito com panos de campo foi colocado sob a região paralombar, elevando-a, de modo a facilitar a visibilidade sonográfica do rim e diminuir a mobilidade do mesmo durante a intervenção. Para aumentar o campo visual sonográfico do rim direito, tendo em vista sua posição anatômica, aplicou-se uma leve pressão na borda caudal da última costela com o polegar, em sentido cranial.

Para a realização do procedimento, o campo foi preparado com solução de iodopovidine alcoólico e foram utilizados materiais estéreis como luvas, aventais, panos de campo, gel acústico estéril, preservativo $^{\mathrm{b}}$ e capa para o transdutor. A rota a ser percorrida pelo cateter foi definida incluindo o trajeto do parênquima renal até a pelve. A distância entre a pele e a pelve renal foi mensurada (Figura 1A), permitindo estimar quanto do comprimento do cateter deveria ser introduzido até atingir o interior da mesma. Caudalmente ao transdutor, foi feita uma pequena incisão cutâtea de $0,5 \mathrm{~cm}$ que facilitou a introdução do instrumento.

Um cateter de modelo pigtaic ( $8 \mathrm{Fr}$ ou $10 \mathrm{Fr}$ ), biocompatível, radiopaco, com orifícios na sua porção distal e ponta helicoidal bloqueadora, foi introduzido 


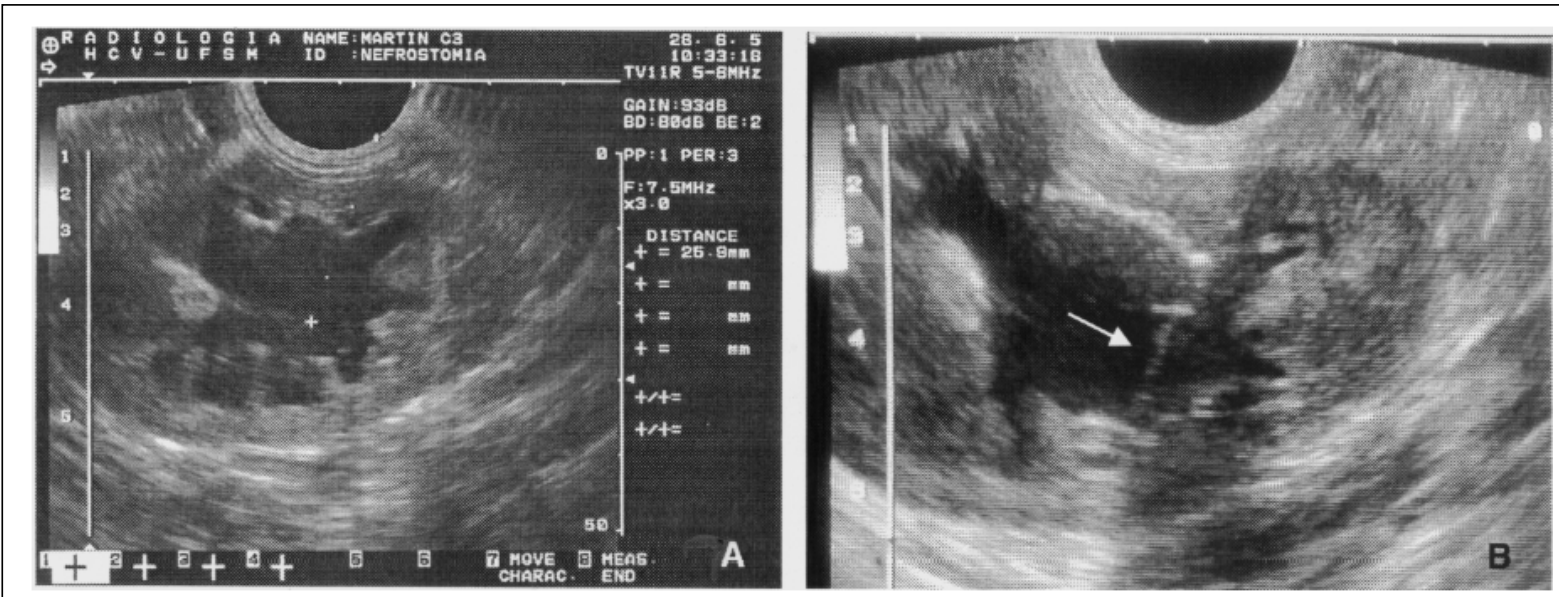

Figura 1 - (A) Sonograma do rim com hidronefrose. Medida da distância entre a pele e o interior da pelve renal (+). (B) Momento em que o cateter atinge a pelve renal dilatada (seta). Inclusão do parênquima renal no percurso (B).

no interior do rim, ecoguiado e sob um ângulo de aproximadamente $45^{\circ}$ em relação ao transdutor, por inserção direta ou através da técnica de Seldinger. A seleção da técnica foi aleatória nos animais, sendo cada uma delas executada duas vezes do lado esquerdo e duas do lado direito, referentes ao rim hidronefrótico.

Na inserção direta, o cateter acoplado ao estilete e à cânula foi introduzido através do parênquima, sob guia ultra-sonográfico, acompanhando-se a ponta do estilete até o interior da pelve (Figura $1 \mathrm{~B}$ ). Ao atingir a mesma, o estilete foi então removido, enquanto a cânula e o cateter foram mantidos na mesma posição dentro do rim.

Na técnica de Seldinger, somente o estilete e a cânula foram inicialmente introduzidos até a pelve renal. A partir daí, o estilete foi retirado para que um fio guia ecogênico e com ponta flexível fosse passado por dentro da cânula, até o interior do rim. Posteriormente, após a remoção da cânula, procedeu-se à ampliação do canal de punção com dilatadores de oito, dez e 12Fr, que foram introduzidos um após o outro, em ordem crescente de calibre, dirigidos pelo fio guia, para que finalmente o cateter fosse inserido.

Em ambos os procedimentos, a porção distal do cateter, rodeada por orifícios, foi deslocada para dentro da pelve de acordo com a profundidade medida pelo ultra-som, sendo, então, completamente removida a cânula ou o fio guia, conforme a técnica empregada. A partir daí foi obtido fluxo livre de urina. Seqüencialmente, a ponta helicoidal do cateter, localizada dentro do rim, foi travada por um mecanismo de tração externo, formado por um fio de náilon, conferindo o bloqueio do mesmo. Para evitar o deslocamento do cateter com o animal em estação, uma porção remanescente do mesmo foi deixada entre o rim e a parede abdominal, antes de fixá-lo à pele com ponto chinês, utilizando náilon monofilamentar 2-0 .

A infusão de meio de contraste à base de diatrizoato sódico e de megluminae diluído a 30\%, através do cateter, opacificou a pelve renal. O volume injetado variou de 5 a $7 \mathrm{ml}$. Radiografias em projeção lateral e ventrodorsal foram obtidas antes e imediatamente após a infusão, para determinar o posicionamento do cateter. Exames ultra-sonográficos de controle foram realizados durante o período em que o dispositivo esteve no rim.

Um recipiente fechado foi conectado ao cateter para que a urina fosse armazenada. Infusões com solução salina estéril ${ }^{\mathrm{f}}$ foram realizadas no mínimo duas vezes ao dia, para lavagem do cateter, durante o período de hematúria. Limpeza do local e troca de bandagens foram feitas diariamente, com intuito de evitar uma possível contaminação. Todos os cães foram mantidos com colar elisabetano, durante o período da nefrostomia. A remoção do cateter e a nefrectomia foram realizadas no segundo ou no terceiro dia após a nefrostomia. Ao término do experimento todos os cães foram encaminhados para adoção.

\section{RESULTADOS E DISCUSSÃO}

Na medicina veterinária, conforme CHRISTIE \& BJORLING (1998), a nefrostomia é indicada para alívio da pressão intra-renal, quando a sua função é evidenciada na urografia excretora. Estes autores, bem como FOSSUM (2005) e HARDIE \& KYLES (2004), descreveram a técnica para colocação de uma sonda de drenagem urinária no interior da pelve renal, por laparotomia. NWADIKE et al. (2000) utilizaram uma incisão abdominal, próxima ao rim, como via de

Ciência Rural, v.37, n.3, mai-jun, 2007. 
acesso para a instalação de cateter. A partir daí, buscouse simplificar os métodos de nefrostomia até então utilizados nos casos de hidronefrose, visando a sua incorporação à rotina veterinária.

O exame ultra-sonográfico localizou facilmente a pelve renal dilatada, sem a necessidade de administração prévia de meio de contraste intravenoso, sendo esse, indispensável à fluoroscopia (STABLES, 1982; ROVEN \& ROSEN, 1984). Em sete cães, a dilatação da pelve renal foi obtida no sétimo dia após a obliteração ureteral, com exceção de um deles, em que a hidronefrose formou-se lentamente, sendo significativa, para permitir o procedimento, somente a partir do $14^{\circ}$ dia após a ligadura do ureter. O exame clínico e laboratorial de uréia e creatinina desse cão permaneceram normais, pela função preservada do rim contralateral.

A medida ecográfica prévia da distância entre a pele e o interior do rim, como sugerido por EARP (2000), permitiu que a introdução do cateter fosse completa, evitando extravasamento de urina pelos orifícios de sua extremidade distal, para o espaço retroperitoneal. Devido à posição anatômica do rim direito nos cães, localizado abaixo do gradil costal, ampliou-se o campo visual sonográfico do mesmo, através da aplicação de uma leve pressão com o polegar na borda caudal da última costela, em sentido cranial. Tal manobra propiciou o adequado posicionamento do transdutor na região paralombar durante o procedimento de nefrostomia percutânea no rim direito.

Nesse trabalho, foram utilizados cateteres de calibres diferentes, que admitiram sua inserção por ambas as técnicas anteriormente descritas. Apesar de ambas as técnicas serem de rápida execução, a técnica de Seldinger mostrou-se mais trabalhosa, tendo em vista a variedade de manobras realizadas previamente à introdução do cateter. Os cateteres de calibre de $8 \mathrm{Fr}$ foram utilizados nos cães com pesos variando de $10 \mathrm{a}$ $13 \mathrm{~kg}$. Os cães com pesos entre 15 e 17,5kg receberam cateteres com calibre de $10 \mathrm{Fr}$, selecionados de forma empírica. Dados a respeito do tamanho do cateter de modelo pigtail, para seu uso em cães, não estão disponíveis na literatura atual.

A pielografia anterógrada percutânea promoveu a opacificação da pelve renal e do ureter e evidenciou, através da interrupção abrupta da coluna de contraste, o ponto de obstrução. A quantidade injetada variou de $5 \mathrm{a} 7 \mathrm{ml}$ e foi equivalente à metade do volume de urina previamente aspirada durante a inserção dos instrumentos. Radiografias em projeção lateral e ventrodorsal foram obtidas (RIVERS et al., 1997), antes (Figura 2 A e B) e imediatamente após a aplicação de contraste (Figura 2 C e D), o que demonstrou o correto posicionamento do cateter dentro da pelve renal (ROVEN \& ROSEN, 1984).

Tanto os exames ultra-sonográficos de controle, quanto a nefrectomia, não demonstraram complicações semelhantes às relatadas na medicina humana, como perfuração de vísceras (WAH et al., 2004), lesão na vasculatura do hilo e hemorragia persistente. O extravasamento de urina para o retroperitônio durante a drenagem urinária (LEWIS \& PATEL, 2004) foi descartado, de acordo com a pielografia anterógrada. Um período transitório de hematúria foi observado logo após a inserção do equipamento perfurante no rim, persistindo por não mais que 24 horas nos oito cães, permanecendo abaixo do limite de tempo relatado em pacientes humanos, de 36 horas (STABLES, 1982).

Frente à acentuada movimentação dos cães, a escolha do cateter de modelo pigtail com extremidade helicoidal e trava (PAUL et al., 2003), associado à fixação da porção remanescente do mesmo, conforme utilizado por EARP (2000) em pacientes humanos obesos, foram fatores determinantes para prevenir o deslocamento do cateter, durante o período de drenagem urinária. No mesmo dia da nefrostomia, após a recuperação da anestesia, os cães apresentaram apetite e comportamento normais. Não houve demonstração de dor na troca de curativo ou mesmo durante os exames de controle ultra-sonográfico e radiográfico contrastado.

\section{CONCLUSÃO}

Baseado nos resultados obtidos no presente estudo, conclui-se que a técnica de nefrostomia percutânea ecoguiada é uma alternativa rápida, segura e eficaz quando aplicada à hidronefrose em cães.

\section{FONTES DE AQUISIÇÃO}

aShimadzu - R. Marquês de São Vicente, 1771. Barra Funda São Paulo, SP.

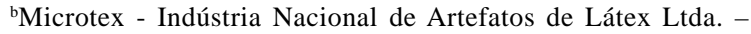
São Roque, SP.

'Skater ${ }^{\circledR}$ Inter. $V^{\mathrm{TM}}$ - PR Hospitalar - Porto Alegre, RS. ${ }^{\mathrm{d}}$ Mononylon 2-0 - Ethicon - São José dos Campos, SP. eUrografina 370 - Shering do Brasil - Santo Amaro, SP. fSolução injetável de Cloreto de Sódio 0,9\% - Ind. Farmacêutica Basa Ltda. - Caxias do Sul, RS.

\section{COMITÊ DE ÉTICA}

Este artigo é produto de um projeto de pesquisa aprovado pela Comissão de Ética e Biossegurança da Universidade Federal de Santa Maria - UFSM/ RS, n. 45/ 2006. 

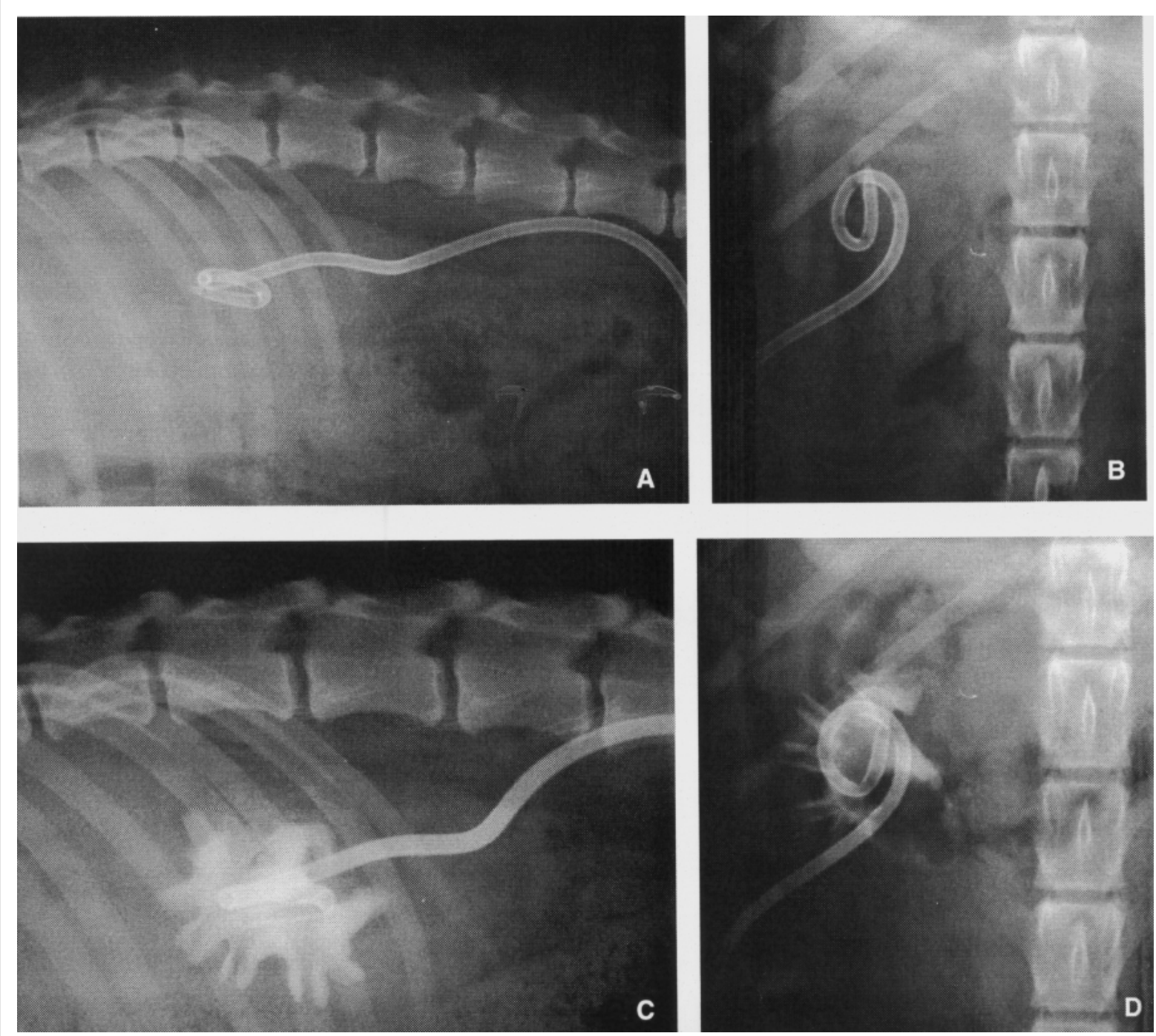

Figura 2 - Radiopacidade do cateter demonstrada por radiografias abdominais simples em projeção lateral (A) e ventrodorsal (B), pós-nefrostomia. Radiografias abdominais em projeção lateral (C) e ventrodorsal (D), após a infusão de meio de contraste, confirmando o posicionamento do cateter na pelve renal e a ausência de extravasamento de urina para o retroperitônio.

\section{REFERÊNCIAS}

BERCOVITCH, M.G. Hydronephrosis. In: TILLEY, L.P.; SMITH, F.W.K. The 5-minute veterinary consult Baltimore: Williams \& Wilkins, 2000. CD-ROM.

CHRISTIE, B.A.; BJORLING, D.E. Rins. In: SLATTER, D. Manual de cirurgia de pequenos animais. São Paulo: Manole, 1998. V.2, cap.104, p.1698-1713.

EARP, P.P. Radiologia intervencionista. Guia prático de urologia, 2000. Cap.7, p.35-39. Capturado em: 26 jan. 2005. On line. Disponível na Internet: <http://www.sbu-mg.org.br/ guia/guia\%20pratico\%20\%20cap\%2007.pdf.>.

FOSSUM, T.H. Cirurgia dos rins e dos ureteres. In: FOSSUM, T.H. et al. Cirurgia de pequenos animais. São Paulo: Roca, 2005. p.551-571.
GUPTA, S. et al. Ultrasound-guided percutaneous nephrostomy in non-dilated pelvicaliceal system. Journal of Clinical Ultrasound, v.26, p.177-179, 1998.

HARDIE, E.M.; KYLES, A.E. Management of ureteral obstruction. Veterinary Clinics North Amer: Small Animal Practice, v.34, p.989-1010, 2004.

HUDSON, J.A. et al. Radiologia abdominal para o clínico de pequenos animais. São Paulo: Roca, 2003. 174p.

KANAYAMA, L.M. Ultra-sonografia intervencionista. In: CARVALHO, C.F. Ultra-sonografia em pequenos animais. São Paulo: Roca, 2004. Cap.23, p.347-358.

LANG, E.K.; PRICE, E.T. Redefinitions of indications for nephrostomy. Radiology, v.147, p.419-426, 1983. 
LEWIS, S.; PATEL, U. Major complications after percutaneous nephrostomy - lesson from a department audit. Clinical Radiology, v.59, p.171-179, 2004.

MATTOON, J.S. et al. Técnicas avançadas e tendências futuras. In: NYLAND, T.G.; MATTOON, J.S. Ultra-som diagnóstico em pequenos animais. São Paulo: Roca, 2005. Cap.19, p.439-454.

McLOUGHLIN, M.A. Surgical emergencies of the urinary tract. Veterinary Clinics of North America: Small Animal Practice, v.30, n.3, p.581-601, 2000.

NWADIKE, B.S. et al. Use of bilateral temporary nephrostomy catheters for emergency treatment of bilateral ureter transection in cat. Journal of the American Veterinary Medical Association, v.217, n.12, p.1862-1865, 2000.

NYLAND, T.G. et al. Biopsia guiada por ultra-som. In: NYLAND, T.G.; MATTOON, J.S. Ultra-som diagnóstico em pequenos animais. São Paulo: Roca, 2005. Cap.3, p.3352.

PAUL, E.M. et al. Choosing the ideal nephrostomy tube. British Journal of Urology International, v.92, p.672677, 2003.

PENNINCK, D.G. Advanced ultrasound techniques. In: NYLAND, T.G.; MATTOON, J.S. Veterinary diagnostic ultrasound. Philadelphia: Saunders, 1995. Cap.14, p.257-258.

RIVERS, B.J. et al. Ultrasonographic-guided, percutaneous antegrade pyelography: technique and application in the dog and cat. Journal of the American Animal Hospital Association, v.33, p.61-68, 1997.

ROVEN, S.J.; ROSEN, R.J. Percutaneous nephrostomy and maintenance of nephrostomy drainage. Special issue to Urology, v.XXIII, n.5, p.25-28, 1984.

SELDINGER, S.I. Catheter replacement of the needle in percutaneous arteriography. Acta Radiologica, v.38, p.368376, 1953.

SOLBIATI, L. New applications of ultrasonography: interventional ultrasound. European Journal of Radiology, v.28, p.200-206, 1998.

STABLES, D.P. Percutaneous nephrostomy: techniques, indications, and results. Urologic Clinics of North America, v.9, n.1, p.15-29, 1982.

SZATMÁRI, V. et al. Ultrasound-guided percutaneous drainage for treatment of pyonephrosis in two dogs. Journal of the American Veterinary Medical Association, v.218, n.11, p.1796-1799, 2001.

THÜROFF, J.W. Percutaneous antegrade endourology. In: TANAGHO, E.A.; McANINCH, J.W. Smith's general urology. Norwalk: Appleton \& Lange, 1988. Cap.8, p.125141 .

WAH, T.M. et al. Percutaneous nephrostomy insertion: outcome data from a prospective multi-operator study at a UK training centre. Clinical Radiology, v.59, p.255-261, 2004. 